\section{Thromboembolism related to a Port-a-Cath device in a patient with cystic fibrosis}

\author{
B Yung, J S Elborn, I A Campbell, \\ Y Summers, M Beckles, A A Woodcock
}

\begin{abstract}
The case is described of a potentially life threatening complication relating to the use of a totally implantable venous access device (Port-a-Cath) in a 28 year old patient with cystic fibrosis. The device was inserted in 1990 and used repeatedly for antibiotic therapy without any complications. In 1995, during assessment for double lung transplantation, a $3 \mathrm{~cm}$ thrombus was found at the tip of the catheter in the right atrium. Embolisation of the thrombus to the pulmonary arteries occurred after the infusion of recombinant tissue plasminogen activator (rt-PA). Thrombus formation may be associated with totally implantable venous access devices and thromboembolism may occur following the use of thrombolytic agents in the treatment of such thrombosis.

(Thorax 1997;52:98-99)
\end{abstract}

Keywords: cystic fibrosis, totally implantable venous access devices, thromboembolism.

Totally implantable venous access devices are used in oncological patients and in patients with cystic fibrosis. ${ }^{12}$ These devices provide safe effective means of intermittent venous access and are well accepted by patients. ${ }^{34} \mathrm{We}$ report a potentially life threatening complication related to the use of a Port-a-Cath device in a patient with cystic fibrosis.

Section of Respiratory Medicine, University of Wales College of Medicine, Llandough Hospital NHS Trust,

Penarth, South Glamorgan CF64 2XX, UK

B Yung

J S Elborn

I A Campbell

North West Lung

Centre, Wythenshawe

Hospital, Manchester

M23 9LT, UK

Y Summers

M Beckles

A A Woodcock

Correspondence to:

Dr B Yung, Department of

Cystic Fibrosis, Royal

Brompton Hospital, London SW3 6NP, UK.

Received 20 June 1995

Accepted for publication

31 July 1995

\section{Case report}

A 28 year old patient with cystic fibrosis (delta F508 heterozygous), who had deteriorated after the birth of her son in 1990, was being assessed for double lung transplantation. She frequently required intravenous antibiotics for chronic Pseudomonas aeruginosa infection of her lungs and venous access became increasingly difficult. In June 1990 a Port-a-Cath was implanted via the right cephalic vein and she subsequently had many antibiotic courses through this device without complication. There was no previous history or family history of thromboembolism and she was not taking the contraceptive pill.

In January 1995 she was referred for assessment for lung transplantation. Her forced expiratory volume in one second $\left(\mathrm{FEV}_{1}\right)$ was 0.761 (26\% predicted) and her body mass

index was $19.1 \mathrm{~kg} / \mathrm{m}^{2}$. Her clotting screen was normal. A routine two dimensional echocardiogram revealed a $3 \mathrm{~cm}$ clot at the catheter tip of the Port-a-Cath in the right atrium. The device was functioning well and the patient was asymptomatic. A bolus dose of $50 \mathrm{mg}$ recombinant tissue plasminogen activator (rt-PA) was given intravenously over an hour on two occasions during the next 16 hours in an attempt to lyse the clot and she was then commenced on intravenous heparin infusion. She remained well until three hours after the last dose of rt-PA when she became acutely unwell and complained of pleuritic chest pain with severe dyspnoea of sudden onset.

On examination she was unwell with tachypnoea and tachycardia. Her blood pressure was $120 / 90 \mathrm{~mm} \mathrm{Hg}$ and oxygen saturation was $92 \%$ on oxygen at $81 / \mathrm{min}$ via nasal cannulae (her normal oxygen saturation was $92 \%$ on air). Her electrocardiogram and chest radiograph were unchanged. A diagnosis of acute pulmonary embolism was made. Her clotting status was checked and a further heparin bolus dose was given because of an inadequate KCCT ratio of 1.05. A computed tomographic (CT) contrast enhanced pulmonary angiogram confirmed the presence of clots in the right main pulmonary artery (fig 1) and the artery to the right lower lobe. A repeat echocardiogram showed no evidence of the clot at the tip of the catheter. The patient's condition was stable and she continued to receive heparin and subsequently warfarin.

Two days later she became more dyspnoeic with production of purulent sputum. Intravenous antibiotics were started and she was also given a $50 \mathrm{mg}$ dose of rt-PA via her Porta-Cath. A repeat CT pulmonary angiogram performed 24 hours later showed persistence of the clots in the pulmonary arteries. She was commenced on streptokinase infusion (250 000 units over 30 minutes) but developed an anaphylactic reaction three minutes into the infusion and the infusion was stopped. Following repeat clotting studies two further doses of rtPA were given over the next six hours but a repeat CT pulmonary angiogram 24 hours later was unchanged. She was then treated with four doses of intravenous urokinase (4400 units/kg on each occasion, given over 30 minutes, with hydrocortisone cover) over the next 18 hours. Her clinical condition improved and a repeat

CT pulmonary angiogram performed 24 hours later revealed resolution of clot in the right main pulmonary artery and in the artery to the right lower lobe. She was discharged three days later on warfarin. A repeat echocardiogram six weeks later revealed no evidence of a recurrence of the clot on the Port-a-Cath which still remains fully functional. Following this case, we electively performed two dimensional echocardiograms on all 11 of our patients with cystic fibrosis with Port-a-Cath devices and no further case of thrombosis was identified.

\section{Discussion}

Port-a-Cath devices have been used in oncological patients since $1982^{1}$ and are as- 
Figure 1 Computed tomographic pulmonary angiogram showing a marked filling defect in the right main pulmonary artery (arrowed).

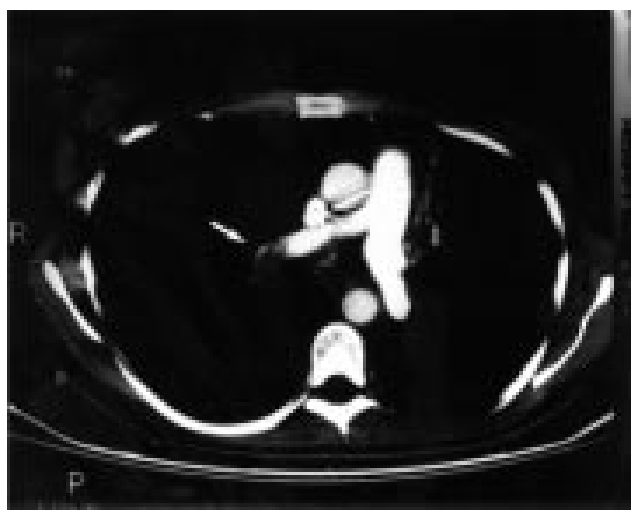

sociated with a lower incidence of infection and thrombosis than external venous access devices, as well as being more cosmetically acceptable. ${ }^{5}$ Pattison reported on the use of the Port-a-Cath in patients with cystic fibrosis in $1986 .^{2}$ Since then, studies have shown that Port-a-Cath devices are well accepted by this group of patients and are safe and reliable for effective intermittent intravenous antibiotic therapy. ${ }^{34}$ Potential complications include catheter occlusion, subcutaneous infiltration, infection, migration of the catheter, pain from the portal and, rarely, venous thrombosis with or without superior vena cava obstruction. ${ }^{346}$ We were able to identify only one previous case of deep venous thrombosis of the iliac veins followed by pulmonary embolism related to the use of a Davol Femoral Infusaport. ${ }^{7}$ Our patient is the first to be reported with thromboembolism related to the use of Port-a-Cath devices implanted in the upper limbs, a serious and potentially life threatening complication. We presume that embolism of the thrombus from the Port-a-Cath occurred following the use of rt-PA.
Thrombolytic agents are effective in the treatment of pulmonary embolism, accelerating clot lysis, hastening pulmonary tissue perfusion, reversing right heart failure, and improving pulmonary capillary blood volume. ${ }^{8}$ They are particularly useful in the treatment of pulmonary embolism when the haemodynamic status is compromised. A serial contrast enhanced helical CT pulmonary angiogram can be used for diagnosis and monitoring the subsequent response to thrombolytic therapy. ${ }^{9}$ We suggest that, in patients with totally implantable venous access devices, thromboembolism should be considered as a differential diagnosis should a patient develop sudden chest pain and dyspnoea, especially if this occurs soon after the infusion of thrombolytic agents or heparin.

BY was supported by the Cystic Fibrosis Trust. We would like to thank staff at the ECG department, Llandough Hospital for performing the echocardiograms.

1 Niederhuber JE, Ensminger W, Gyves JW, Liepman M, Doan $\mathrm{K}$, Cozzi E. Totally implanted venous and arterial acces system to replace external catheters in cancer treatment. Surgery 1982;92:706-11

2 Pattison J, Heaf DP. Totally implantable vascular access in treatment of cystic fibrosis. Lancet 1986;i:799.

3 Cassey J, Ford WDA, O'Brien L, Martin AJ. Totally implantable system for venous access in children with cystic fibrosis. Clin Pediatr 1988;27:91-5.

4 Morris JB, Occhionero ME, Gauderer MWL, Stern RC, Doershuk CF. Totally implantable vascular access devices in cystic fibrosis: A four year experience with fifty eight patients. F Pediatr 1990;117(1 Pt 1):82-5.

5 Brothers TE, Von Moll LK, Niederhuber JE, Roberts JA, Walker-Andrews S, Ensminger WD. Experience with JA, Walker-Andrews S, Ensminger WD. Experience with subcutaneous infusion ports in three hundred patients. Surgery

6 Peckham DG, Hill J, Manhire AR, Knox AJ. Resolution of superior vena cava obstruction following thrombolytic therapy in a patient with cystic fibrosis and a long-term indwelling catheter. Respir Med 1994;88:627-9.

7 Sola JE, Stone MM, Wise B, Colombani PM. Atypical thrombotic and septic complications of totally implantable venous access devices in patients with cystic fibrosis. Pediatr Pulmonol 1992;14:239-42.

8 Goldhaber SZ. Contemporary pulmonary embolism thrombolysis. Chest 1995;107(Suppl 1):45-51S.

9 Curtin JJ, Mewissen MW, Crain MR, Lipchik RJ. Post contrast $\mathrm{CT}$ in the diagnosis and assessment of response to thrombolysis in massive pulmonary embolism. f Comput Assist Tomogr 1994:18:133-5.

\section{Transverse myelitis: a reversible complication of bronchial artery embolisation in cystic fibrosis}

K L Fraser

R H Hyland

D E Tullis

\section{Department of \\ Radiology}

H Grosman

The Wellesley Hospital, University of Toronto,

Toronto, Ontario M4Y

1JS, Canada

Correspondence to:

Dr D E Tullis.

Received 14 December 1995

Returned to authors

29 March 1996

Revised version received

12 August 1996

Accepted for publication

3 October 1996
K L Fraser, H Grosman, R H Hyland, D E Tullis

\section{Abstract}

The case history is presented of a young woman with cystic fibrosis and life threatening haemoptysis. Angiography revealed enlarged bronchial vessels, one of which supplied the contralateral lung. Trans- verse myelitis developed following bronchial artery embolisation but recovery was rapid and nearly complete. Haemoptysis did not recur during four years of follow up.

(Thorax 1997;52:99-101)

Keywords: cystic fibrosis, bronchial artery, embolisation, transverse myelitis.

A 28 year old woman who was diagnosed with cystic fibrosis in infancy had moderate lung disease (forced vital capacity $75 \%$ predicted, forced expiratory volume in one second $61 \%$ predicted) and was colonised with Pseudomonas aeruginosa and Burkholderia cepacia. She was generally well with few hospital admissions for pulmonary exacerbations. In July 1991 she developed haemoptysis associated with a pulmonary exacerbation, resulting in a significant fall in her haemoglobin level. She improved with conservative treatment.

Haemoptysis recurred in October 1991 and her haemoglobin fell from $13.4 \mathrm{~g} / \mathrm{dl}$ to $11.4 \mathrm{~g} / \mathrm{dl}$. 
Figure 1 Injection of right fifth intercostal (solid black arrow) with remote filling of abnormally hypertrophied nonbronchial systemic artery (open arrow) supplying the right upper lobe.

Figure 2 Enlargement of abnormally hypertrophied vessel shown in fig 1.
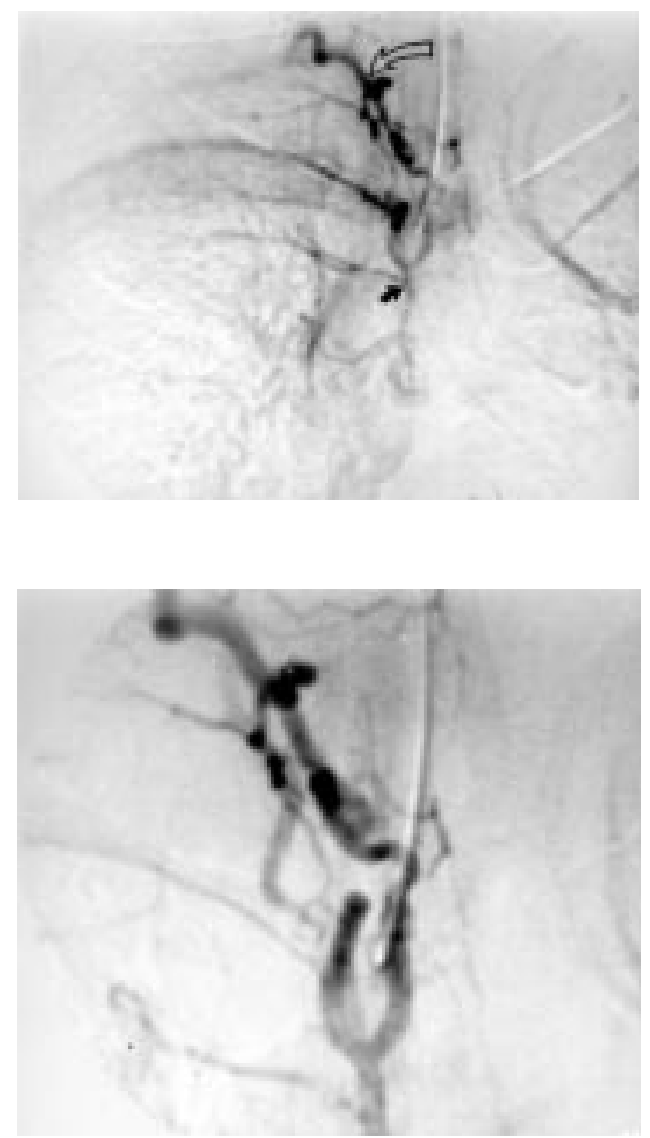

Figure 3 Abnormal nonbronchial systemic artery successfully embolised (black arrow). Stasis of contrast medium in residual right fifth intercostal artery (black arrowheads) with communicating branches with other intercostal vessels and no visualisation of vascular supply to the spinal cord.
The right fifth intercostal artery, when injected with contrast, filled more proximally positioned intercostal vessels through communicating branches parallel to the spine until eventually filling an abnormally enlarged nonbronchial systemic artery supplying the right upper lobe. This large abnormal vessel could only be embolised remotely via the right fifth intercostal artery. The catheter became wedged into this vessel during the lengthy embolisations and there was angiographic "hold up" or stagnation of contrast medium in the proximal intercostal vessels on "control" injections into the right fifth intercostal artery (figs $1-3$ ).

The left thyrocervical trunk gave rise to an enlarged bronchial vessel which crossed the midline and supplied the right lower lobe (a rare congenital anomaly). Since this was a very large vessel, it was embolised with a combination of Ivalon and absorbable gelatin sponge particles (Gelfoam; Upjohn, Ontario, Canada). A fourth enlarged bronchial artery arising from the right thyrocervical trunk and supplying the right mid lung was cannulated and embolised with the same combination.

A 5 French end hole embolisation Cobra catheter (Cook Inc, Bloomington, Indiana, USA) was used for the first two vessels embolised and a 5 French $\mathrm{HIH}$ (head hunter) catheter (Cook Inc) for the enlarged bronchial arteries arising from each of the thyrocervical trunks. The procedure lasted approximately four hours and a total of $300 \mathrm{ml}$ of non-ionic contrast medium was injected. Digital subtraction angiography was used entirely during the procedure in an attempt to keep the volume of contrast medium used to a minimum and to assess better the vascular supply to the cord. No obvious vascular supply to the cord, however, was visualised during the entire study. One limitation might have been the lack of a high resolution digital system although the patient was anaesthetised, thus eliminating respiratory motion artefact during the digital subtraction imaging. The contrast medium used was non-ionic (Omnipaque 300; SanofiWinthrop, Ontario, Canada) which is considered safer than ionic based media. Injections into each vessel of $2-4 \mathrm{ml}$ contrast medium per injection were carried out manually by the angiographer.

All four abnormal vessels were successfully

She required a total of four units of blood. Conservative treatment was not successful and three days after admission she was scheduled for bronchial arteriography and embolisation. On the morning of the scheduled procedure she had massive haemoptysis with respiratory compromise necessitating intubation. Bronchial artery angiography was performed. The first artery recognised as a bronchial artery arose from the left side of the aorta at around T7. As embolisation was performed using polyvinyl alcohol microspheres 300-600 $\mu \mathrm{m}$ (Ivalon; Locam Medical Distributors, Laboratories Nycamed, Paris, France) more and more enlarged collateral vessels supplying the left lung became apparent and were further embolised. embolised. The haemoptysis stopped, the patient was extubated and reported no neurological symptoms for the remainder of the day. The following morning the patient complained of paralysis of the left leg and was found to have right sided sensory disturbance to the level of T4. Posterior column function was intact, compatible with a diagnosis of transverse myelitis.

Treatment was initiated immediately with intravenous methylprednisolone $125 \mathrm{mg}$ daily improvement. At the time of discharge 25 days later some sensory deficit and minimal foot drop persisted. With rehabilitation therapy no discernible motor weakness remained, although neurological examination revealed refor three days and there was rapid neurological 
duced sensation to pin prick and light touch on the right to the level of the umbilicus.

The patient remained well with no recurrent massive haemoptysis until August 1995 when she succumbed to an infective exacerbation of her pulmonary disease.

\section{Discussion}

The bronchial artery circulation is quite variable and complex. Arterial origins arise mainly from the thoracic aorta or its branches. Up to $20 \%$ arise from various other vessels and the remaining $10 \%$ originate from the anterior surface of the aortic arch. In conditions of chronic inflammation such as cystic fibrosis and tuberculous bronchiectasis, the bronchial circulation expands greatly. ${ }^{1}$ The same vessels that supply the bronchial arteries may also supply the oesophagus, mediastinal lymph nodes, and the spinal arteries through a complex anastomotic network. This expanded systemic circulation is the primary source of bleeding in haemoptysis. ${ }^{2}$ Many patients with cystic fibrosis have undergone bronchial artery embolisation for treatment of haemoptysis. ${ }^{3-6}$ We are aware of one previous case in which an hypertrophied bronchial vessel has supplied the contralateral lung. ${ }^{7}$ In that report, the patient had undergone multiple previous embolisations and the authors hypothesised that the previous interventions may have contributed to the enlargement of the vessel. Our patient had no previous history of embolisation, suggesting that this uncommon circulation may arise simply as a result of chronic inflammation.

Transverse myelitis is a well recognised complication of arteriography ${ }^{89}$ and is a theoretical risk of bronchial artery embolisation because of the anatomical variation of a shared origin of bronchial arteries with intercostal vessels which supply radiculomedullary branches to the anterior spinal circulation. However, there are no reports in the literature of transverse myelitis following bronchial artery embolisation. Kardjiev et al reported five cases in which similar neurological events followed the injection of contrast medium into the right fifth intercostal artery. The procedures were carried out for diagnostic purposes only and a different contrast medium (ionic) from ours was used. All patients made a full recovery although it took almost three months for one patient's symptoms to resolve. ${ }^{10}$
Our patient is similar in that contrast material was injected into the fifth right intercostal artery and in the subsequent neurological course. Given the delayed onset of the neurological event and the reversible nature of the lesion, and given the lack of visualisation of the anterior spinal artery complex and the amount of embolic material used, we postulate that this, too, is a case of a contrast-induced spinal cord lesion. This may have been promoted by the wedging of the catheter into the right fifth intercostal origin and the persistence of contrast medium in this vessel and its branches during this lengthy, although life saving, procedure.

The anterior spinal cord is supplied by the anterior spinal artery which originates from branches of the vertebral arteries and from anterior medullary branches of intercostal and lumbar arteries. ${ }^{11}$ All spinal cord arteries are functional end arteries. In the thoracic area the supply to the anterior spinal artery is usually from a single anterior medullary branch. It has been suggested that, in patients who suffer neurological sequelae following injection of contrast medium, the fifth right intercostal artery sometimes gives rise to the anterior vascular supply of the thoracic cord at T4 to T6. ${ }^{10}$ One cannot predict ahead of time in which patient this occurs.

This case illustrates that transverse myelitis is a risk of bronchial artery embolisation, even when current guidelines and proper angiographic procedures are followed. However, the prognosis for a nearly total recovery is good.

1 Deffebach ME. Clinical relevance of the bronchial circulation. Pulm Perspect 1991;8:8-10.

2 Porter DK, VanEvery MJ, Anthracile RF, Mack JW. Massive hemoptysis in cystic fibrosis. Arch Intern Med 1983;143 287-90.

3 Fellows KE, Khaw KT, Schuster S, Shwachman H. Bronchial artery embolization in cystic fibrosis: technique and long-term results. $\mathcal{F}$ Pediatr 1979;95:959-63.

4 Sweezey NB, Fellows KE. Bronchial artery embolization for severe hemoptysis in cystic fibrosis. Chest 1990;97: 1322-6.

5 Cohen AM, Doershuk CF, Stern RC. Bronchial artery embolization to control hemoptysis in cystic fibrosis. Radiology 1990;175:401-5.

6 Tonkin ILD, Hanissian AS, Boulden TF, et al. Bronchia arteriography and embolotherapy for hemoptysis in patients with cystic fibrosis. Cardiovasc Intervent Radiol 1991;14:241-6.

7 Cohen AM, Antoun BW, Stern RC. Left thyrocervical trunk bronchial artery supplying right lung: source of recurrent hemoptysis in cystic fibrosis. AfR 1992;158:1131-3.

8 Feigelson HH, Ravin HA. Transverse myelitis following selective bronchial arteriography. Radiology 1965;85:663-

9 DiChiro G. Unintentional spinal cord arteriography: a warning. Radiology 1974;112:231-3.

10 Kardjiev V, Symeonov A, Chankov I. Etiology, pathogenesis and prevention of spinal cord lesion in selective angiography of the bronchial and intercostal arteries. Radiology 1974;112:81-3.

11 Stoll JF, Bettmann MA. Bronchial artery embolization to control hemoptysis: a review. Cardiovasc Intervent Radio 1988;11:263-9. 